Paraxial diffraction on structures generated by multiplicative iterative procedures

This content has been downloaded from IOPscience. Please scroll down to see the full text.

2003 J. Opt. A: Pure Appl. Opt. 5 S324

(http://iopscience.iop.org/1464-4258/5/5/386)

View the table of contents for this issue, or go to the journal homepage for more

Download details:

IP Address: 147.96.14.16

This content was downloaded on 30/01/2014 at 17:50

Please note that terms and conditions apply. 


\title{
Paraxial diffraction on structures generated by multiplicative iterative procedures
}

\author{
Tatiana Alieva and Maria Luisa Calvo \\ Departamento de Óptica, Facultad de Ciencias Físicas, Universidad Complutense de Madrid, \\ 28040 Madrid, Spain
}

E-mail: talieva@fis.ucm.es and mlcalvo@fis.ucm.es

Received 14 November 2002, accepted for publication 13 June 2003

Published 22 August 2003

Online at stacks.iop.org/JOptA/5/S324

\begin{abstract}
We consider Fresnel and Fraunhofer diffraction on regular structures generated by multiplicative iterative procedures. We prove that the iterative procedure of field construction is preserved during Fresnel diffraction, while the initiator of the structure in the Fresnel region is the Fresnel transform of the original structure. The diffractive field in the Fraunhofer region is quasi-periodic. The theoretical results are proved experimentally.
\end{abstract}

Keywords: Paraxial diffraction, Fourier optics, fractals, multiplicative gratings

\section{Introduction}

Over the last decade the concept of fractal geometry, introduced by Mandelbrot [1], has been explored in diverse areas of science, including optics. Thus, exciting experiments on light scattering and diffraction by fractal objects have been performed and explained theoretically [2-8]. Moreover, it was shown that some optical fields have fractal structure themselves. So, after propagation of a coherent plane wave through a periodic grating with sharp-edged slits, the field observed at a distance equal to the product of an irrational number and the Talbot distance has fractal properties [9]. Another example of fractal fields is the fractal structure of the eigenmodes of unstable-cavity lasers [10]. Research on wave interactions with fractal objects and the discovery of fractal features of electromagnetic fields has led to the development of fractal electrodynamics and fractal optics. Nevertheless, consideration of diffraction by fractal gratings is mostly restricted to the well known regular fractal objects, such as Cantor sets, Koch curves, Sierpinski's carpet etc [3-8].

A typical property of fractal objects is that they are constructed by iterative procedures. In this paper we consider the peculiarities of paraxial diffraction (in the Fresnel and Fraunhofer regions) on the regular structures generated by multiplicative iterative procedures which, in particular cases, correspond to the fractal and to the periodic gratings. Note that not only the amplitude but also the phase or complex amplitude gratings can be represented this way.

\section{Structures generated by multiplicative iterative procedures}

The gratings generated by multiplicative procedures can be described fully by the complex transmittance initiator function $F_{0}(x)$, the size of the elementary cell $\boldsymbol{a}=\left(a_{x}, a_{y}\right)$, and the generator $f_{1}$, represented by matrix $M_{x} \times M_{y}$, which characterises the translation rule of the initiator. Here, for simplicity, we will consider the one-dimensional case and therefore $f_{1}=\left[f_{1}(0), f_{1}(1), \ldots, f_{1}(M-1)\right]$. Moreover, we suppose that an initiator $F_{0}(x)$ is defined in the onedimensional interval $x \in[0, a]$ in order to simplify the description of the transmittance function of the entire grating. A grating of level $n$, constructed through the multiplicative iterative procedure, is defined as

$$
\begin{aligned}
F_{n}(x) & =\sum_{m=0}^{M-1} f_{1}(m) F_{n-1}(x) * \delta\left(x-m M^{n-1} a\right) \\
& =\sum_{m=0}^{M-1} f_{1}(m) F_{n-1}\left(x-m M^{n-1} a\right),
\end{aligned}
$$

where $*$ stands for the convolution operation. In particular, the grating of level 1 is given by $F_{1}(x)=\sum_{m=0}^{M-1} f_{1}(m) F_{0}(x-m a)$. Note that, in general, the signal $F_{n}(x)$ is a complex function, because the initiator as well as the generator matrix can take complex values.

Such types of multiplicative cascades for real non-negative values of the generator matrix produce a variety of well 
Paraxial diffraction on structures generated by multiplicative iterative procedures

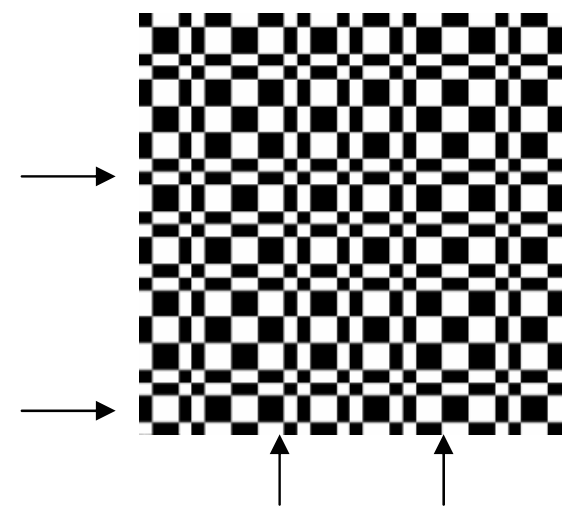

Figure 1. A multiplicative grating of level 5, constructed using a generator $f_{1}=[1,-1 ;-1,1]$.

known regular fractals. Thus, when the initiator is a bar and the generator is $f_{1}=[1,0,1]$, the triadic Cantor set is obtained. Meanwhile, the generator $f_{1}=[p, q]$, where $p$ and $q$ are positive numbers $(p+q=1)$, produces the binomial multiplicative measures [1]. The generator with equal elements $\left(f_{1}(m)=1\right)$ corresponds to periodic objects of $M^{n}$ periods. In the context of this paper, we will call these structures finite periodic structures. The twodimensional generators $\boldsymbol{f}_{1}=[1,0,1 ; 0,1,0 ; 1,0,1]$ and $\boldsymbol{f}_{1}=$ $[1,1,1 ; 1,0,1 ; 1,1,1]$, where the initiator is a square, lead to the Vicsek fractal and the Sierpinski carpet, respectively. Moreover, we can enlarge the class of structures if the negative or complex values of the generator matrix are considered. Thus one can use the $2 \times 2$ Hadamard matrix $f_{1}=[1,1 ; 1,-1]$ as a generator. The grating used for our diffraction experiments (see figure 1) is obtained from the square $a \times a$ as an initiator and $f_{1}=[1,-1 ;-1,1]$ as the generator after $N=5$ iterations. Note that the lighter colour reproduces the negative values. We used this substitution to be able to work with an amplitude grating.

The discrete signals obtained through the multiplicative procedures and their Fourier spectra have been studied in $[11,12]$. We can generalize these results for the case of multiplicative gratings. Thus, introducing a row vector $f_{n}=\left[f_{n}(0), f_{n}(1), \ldots, f_{n}\left(M^{n}-1\right)\right]$ of level $n$ constructed from the generator vector $f_{1}$ such that

$$
f_{n}=\left[\begin{array}{c}
{\left[f_{n-1}\right]^{\mathrm{t}} f_{1}(0)} \\
{\left[f_{n-1}\right]^{\mathrm{t}} f_{1}(1)} \\
\cdots \\
{\left[f_{n-1}\right]^{\mathrm{t}} f_{1}(M-1)}
\end{array}\right]^{\mathrm{t}},
$$

one can represent the multiplicative grating in the form

$$
\begin{aligned}
F_{n}(x) & =\sum_{m=0}^{M^{n}-1} f_{n}(m) F_{0}(x) * \delta(x-m a) \\
& =\sum_{m=0}^{M^{n}-1} f_{n}(m) F_{0}(x-m a)
\end{aligned}
$$

It is easy to see that decimation of the vector of level $n$ produces the vector of the lower level

$$
f_{n}\left(k+M^{n-1} m\right)=f_{1}(m) f_{n-1}(k),
$$

and therefore

$$
F_{n}\left(x+M^{n-1} m a\right)=f_{1}(m) F_{n-1}(x) .
$$

Iterating equation (4), we derive that the $j$ th element of vector $f_{n}$ can be written in the form

$$
f_{n}(j)=\prod_{s=0}^{n-1} f_{1}\left(m_{s}\right)
$$

where

$$
j=m_{0}+m_{1} M+\cdots+m_{l} M^{l}+\cdots+m_{n-1} M^{n-1}
$$

and $m_{s}=0, \ldots, M-1(s=0, \ldots, n-1)$. This means that the multiplicative vector $f_{n}$ can be represented as an elementby-element product of $n$ finite periodic vectors $p_{k}$ :

$$
\boldsymbol{f}_{n}=\prod_{k=1}^{n} \boldsymbol{p}_{k}
$$

constructed as

$$
\boldsymbol{p}_{k}=[\underbrace{\boldsymbol{v}_{k}, \boldsymbol{v}_{k}, \ldots, \boldsymbol{v}_{k}}_{M^{n-k}}]
$$

where

$$
\begin{aligned}
\boldsymbol{v}_{k}= & {[\underbrace{f_{1}(0), \ldots, f_{1}(0)}_{M^{k-1}}, \underbrace{f_{1}(1), \ldots, f_{1}(1)}_{M^{k-1}}, \ldots,} \\
& \underbrace{f_{1}(M-1), \ldots, f_{1}(M-1)}_{M^{k-1}}] .
\end{aligned}
$$

The following table helps us understand the generating procedure of vector $f_{n}$, represented by equations (2), (6) and (8), for the case $M=2, n=3$ :

$$
\begin{array}{llllllll}
f_{1}(0) & f_{1}(1) & f_{1}(0) & f_{1}(1) & f_{1}(0) & f_{1}(1) & f_{1}(0) & f_{1}(1) \\
f_{1}(0) & f_{1}(0) & f_{1}(1) & f_{1}(1) & f_{1}(0) & f_{1}(0) & f_{1}(1) & f_{1}(1) . \\
f_{1}(0) & f_{1}(0) & f_{1}(0) & f_{1}(0) & f_{1}(1) & f_{1}(1) & f_{1}(1) & f_{1}(1)
\end{array}
$$

Every $k$ th row, in according with equation (9), is a finite periodic vector with $M^{n-k}=2^{3-k}$ periods. By multiplying the two first rows element-by-element, the vector $\left[f_{2}, f_{2}\right]$ is obtained. Then, after multiplication on the third row, the vector $\boldsymbol{f}_{3}$ is constructed: $\boldsymbol{f}_{3}=\left[f_{1}(0) \boldsymbol{f}_{2}, f_{1}(1) \boldsymbol{f}_{2}\right]$, which corresponds to equation (2).

The multiplicative grating with initiator $F_{0}(x)$ that is limited by the cell size $a\left(F_{0}(x)=0\right.$, for $\left.x \notin[0, a]\right)$ can be represented correspondingly as a product of $n+1$ finite periodic gratings, constructed the following way. The first grating $T_{0}(x)$ is finite and periodic, with $M^{n}$ periods, and is obtained from the initiator $F_{0}(x)$ in accordance with equation $(3)\left(f_{1}(m)=1\right)$ :

$$
T_{0}(x)=\sum_{m=0}^{M^{n}-1} F_{0}(x) * \delta(x-m a) .
$$

Next, grating $P_{1}$ is periodic, with $M^{n-1}$ periods, and is constructed from the rectangular function $R(x)$ (defined in the interval $x \in[0, a])$ and vector $\boldsymbol{v}_{1}=\boldsymbol{f}_{1}$ as

$$
P_{1}(x)=\sum_{j=0}^{M^{n-1}} \sum_{m=0}^{M-1} R(x) v_{1}(m) * \delta(x-m a) * \delta(x-j a)
$$


In general, the $k$ th grating $P_{k}$ is periodic, with $M^{n-k}$ periods, and is defined by the same rectangular function $R(x)$ and vector $\boldsymbol{v}_{k}$ as

$$
P_{k}(x)=\sum_{j=0}^{M^{n-k}-1} \sum_{m=0}^{M^{k}-1} R(x) v_{k}(m) * \delta(x-m a) * \delta(x-j a) .
$$

Note that $P_{n}$, the last grating in this series, is not really periodic because it contains only one period. Moreover, we observe that the grating $P_{k}$ is obtained from the grating $P_{1}$ by the coordinate scaling $P_{k}(x)=P_{1}\left(x / M^{k-1}\right)$. Finally, the multiplicative grating can be represented as a product of the form factor $T_{0}(x)$, defined by the initiator $F_{0}$, and the structure factor, defined by the generator $f_{1}$ :

$$
F_{n}(x)=T_{0}(x) \prod_{k=1}^{n} P_{k}(x)=T_{0}(x) \prod_{k=1}^{n} P_{1}\left(x / M^{k-1}\right) .
$$

Similar presentations of some fractal gratings such as Cantor bars, the Sierpinski carpet and Sierpinski gasket have been used for the analysis of Fraunhofer diffraction in [8]. Since the initiator of these fractals is a rectangular or square function for one- or two-dimensional structures correspondingly, the additional multiplication on the grating $T_{0}(x)$ has not been used in [8]. Note that representation (14) of the multiplicative gratings defined by equation (1) is possible only if the initiator function $F_{0}(x)$ is zero outside the elementary cell of size $a$.

\section{Fraunhofer diffraction on multiplicative gratings}

Let us consider Fraunhofer diffraction of a plane wave on a multiplicative grating. The complex field amplitude in the Fraunhofer region is described through the Fourier integral of the input. Here we use dimensionless variables. Thus, in the one-dimensional case, for the Fourier transform (FT) of the multiplicative structure $F_{n}(x)$, which is defined by equation (1), we obtain

$$
\begin{aligned}
G_{n}(u) & =\int_{-\infty}^{\infty} F_{n}(x) \exp (-\mathrm{i} 2 \pi x u) \mathrm{d} x \\
& =G_{n-1}(u) \sum_{m=0}^{M-1} f_{1}(m) \exp \left(-\mathrm{i} 2 \pi u m M^{n-1} a\right) \\
& =G_{0}(u) \prod_{j=1}^{n} \sum_{m=0}^{M-1} f_{1}(m) \exp \left(-\mathrm{i} 2 \pi u m M^{j-1} a\right),
\end{aligned}
$$

where $G_{0}(u)$ is the FT of initiator $F_{0}(x)$. As is usual for fractal structures [3], the FT of the multiplicative signal is divided into two parts: the form and structure factors:

$$
G_{n}(u)=G_{0}(u) S_{n}(u) .
$$

The form factor $G_{0}(u)$ is defined by the FT of the initiator; the structure factor $S_{n}(u)$ reveals the iterative procedure of the grating construction. By introducing a periodic function $H_{j}(u)$ with period $\left(M^{j-1} a\right)^{-1}$,

$$
H_{j}(u)=\sum_{m=0}^{M-1} f_{1}(m) \exp \left(-\mathrm{i} 2 \pi u m M^{j-1} a\right)=H_{1}\left(u M^{j-1}\right),
$$

we derive that the structure factor of the FT of a multiplicative signal of level $n$ can be written as either a product of $n$ periodic functions $H_{j}(u)(j=1,2, \ldots, n)$ with frequencies $M^{j-1} a$ or a product of $n$ scaled replicas of the structure factor of the first level $H_{1}(u)$ :

$$
S_{n}(u)=\prod_{j=1}^{n} H_{j}(u)=\prod_{j=1}^{n} H_{1}\left(u M^{j-1}\right) .
$$

Alternatively, if $F_{n}(x)$ in the form (3) is introduced in the Fourier integral, then the structure factor can be represented by the Fourier series

$$
S_{n}(u)=\sum_{m=0}^{M^{n}-1} f_{n}(m) \exp (-\mathrm{i} 2 \pi u m a),
$$

which clearly indicates its periodicity with period $a^{-1}$.

In the particular case of the periodic gratings $\left(f_{1}(m)=1\right)$, the structure factor $S_{n}(u)$ can be written as

$$
\begin{aligned}
S_{n}(u) & =\sum_{m=0}^{M^{n}-1} \exp (-\mathrm{i} 2 \pi u m a) \\
& =\exp \left[-\mathrm{i} \pi u a\left(M^{n}-1\right)\right] \frac{\sin \left(\pi u M^{n} a\right)}{\sin (\pi u a)} .
\end{aligned}
$$

We observe that the structure factor of the finite periodic gratings is a periodic function with period $a^{-1}$. For the case where $M$ or $n$ goes to infinity, $S_{n}(u)$ transforms to a comb function.

Comparing equation (14) with (16) and (18), we observe a similarity in the construction of the multiplicative grating itself and its FT. Indeed, the structure factors in both cases are constructed as a product of $n$ scaled replicas of the periodic signals (for the case of the grating, these signals have finite support) with scaling coefficients $M^{j-1}$ for $j=1, \ldots, n$. The form factors in both cases are defined only by the initiator function $F_{0}(x)$. Nevertheless, such representation of the FT is valid for all multiplicative gratings described by equation (1), even if the initiator $F_{0}(x)$ is spread outside the elementary cell, which is not the case for the validation of relationship (14).

In figure 2(a), which displays the experimental Fourier spectrum of the grating represented in figure 1, the quasiperiodic behaviour of $S_{n}$ can be observed, slightly masked by the form factor. Thus, in figure $2(b)$, we show part of the spectrum-indicated by the white box in figure $2(a)$ and enlarged by a factor of two-which is similar to certain parts of the whole spectrum. In our experiments we used an HeNe laser (wavelength $\lambda=632.8 \mathrm{~nm}$ ), a Fourier lens of focal distance $50 \mathrm{~cm}$, and a Hitachi KP-140 CCD camera. The size of the initiator was $a=0.49 \mathrm{~mm}$.

\section{Fresnel diffraction on multiplicative gratings}

The Fresnel diffraction of a plane wave on a grating is described mathematically as a convolution of the grating transmittance function $g(x)$ and the quadratic chirp function $W(x, z)=g(x) *(\mathrm{i} z)^{-1 / 2} \exp \left(\mathrm{i} \pi x^{2} / z\right)$, where $z=\lambda d, \lambda$ is the wavelength, and $d$ is the distance from the object plane. It has been proved in various articles (see, for example, [5-7]) that, for objects with a scaling symmetry $g(x / m)=m^{\alpha} g(x)$ (which 


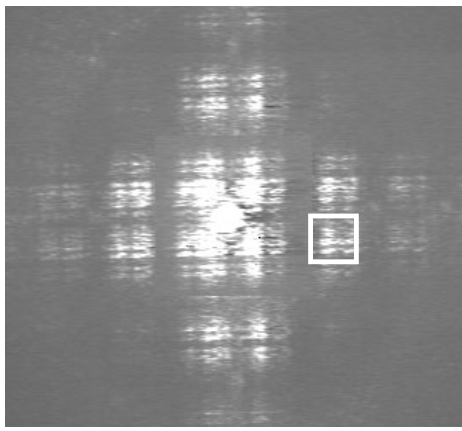

$a$

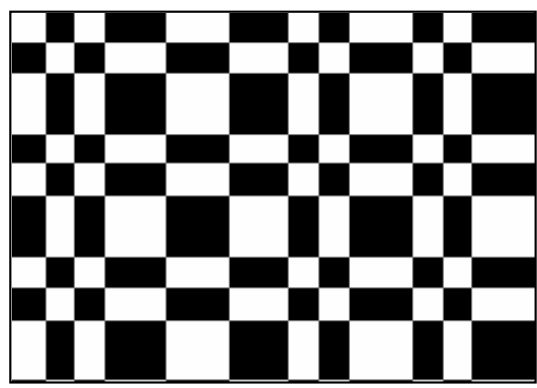

$a$

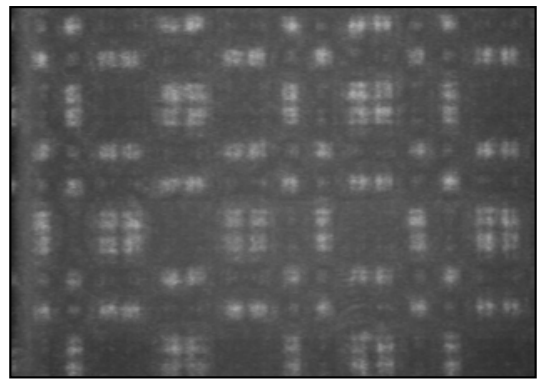

$b$

Figure 3. (a) The intensity distribution of part of the original multiplicative grating (indicated by arrows in figure 1) and its initiator, enlarged by a factor of two. (b) The intensity distribution of the Fresnel pattern of the multiplicative grating at a distance $d=23.6 \mathrm{~cm}$ and its initiator (the Fresnel transform of the initiator of the original grating), enlarged by a factor of two.

possible to approximate equation (23) in the form (14), where $W_{0}(x, z)$ is used instead of $F_{0}(x)$. Moreover, in this case, the intensity distributions of the Fresnel patterns $I_{n}(x, z)$ are also constructed through the multiplicative iterative procedure:

$$
\begin{aligned}
I_{n}(x, z) & =\left|W_{n}(x, z)\right|^{2} \\
& =\left|\sum_{m=0}^{M-1} f_{1}(m) W_{n-1}\left(x-m M^{n-1} a, z\right)\right|^{2} \\
& \approx \sum_{m=0}^{M-1}\left|f_{1}(m)\right|^{2} I_{n-1}\left(x-m M^{n-1} a, z\right) \\
& =\sum_{m=0}^{M-1} t_{1}(m) I_{n-1}\left(x-m M^{n-1} a, z\right),
\end{aligned}
$$

where generator $\boldsymbol{t}_{1}=\left[\left|f_{1}(0)\right|^{2},\left|f_{1}(1)\right|^{2}, \ldots,\left|f_{1}(M-1)\right|^{2}\right]$ and the initiator is $\left|W_{0}(x, z)\right|^{2}$.

Figure 3 shows (a) the intensity distributions of part of the original grating, indicated by the arrows in figure 1, and (b) the corresponding Fresnel diffraction pattern for a distance $d=23.6 \mathrm{~cm}\left(\pi a^{2} / d \lambda \approx 5\right)$ and the intensity distributions of their initiators, enlarged by a factor of two. As we can see, for small $d$ the intensity distribution satisfies relationship (25). For larger $d$, the initiator of the multiplicative field in the Fresnel region $W_{0}(x, z)$ is spread out of the elementary cell and the intensity diffraction patterns cannot be approximated by equation (25).
Note that, even if the expression (14) is valid for the grating itself, the corresponding relationship in general does not hold for the diffractive fields because, due to the diffraction, $W_{0}(x, z)$ is spread out of the elementary cell. Only for relatively small distances (where the initiator $W_{0}(x, z)$ is confined to the same region $a$ as the initiator $F_{0}(x)$ ) might it be 


\section{Conclusions}

Two important properties of the paraxial diffraction of a plane wave on multiplicative gratings have been discussed: the quasi-periodicity of the field in the Fraunhofer region and the invariance of the iterative procedure of field construction during Fresnel diffraction. In has been shown that Fresnel diffraction of the plane wave on the multiplicative grating produces iterative, fractal-like field structure, with a complex initiator that is the Fresnel transform of the initiator of the original grating. The characteristics of these multiplicative fields can not always be determined from an analysis of their intensity patterns.

Since only the convolution property of the Fresnel integral has been used to obtain relationship (23), we can conclude that another convolution-type transformation of a multiplicative function also preserves the iterative procedure of its construction. In this case, a new initiator will be the result of the corresponding transform of the initiator $F_{0}(x)$. It means that, by performing the convolution-type filtering operation of the multiplicative field, we obtain a multiplicative field constructed by the same iterative procedure.

The redundancy of multiplicative gratings (where a similar information-carrying structure repeats in different parts of the grating) leads to redundancy of the corresponding diffractive fields (in the Fraunhofer and Fresnel regions), which may be used for optical communication.

\section{Acknowledgment}

This work was supported partially by the Spanish Ministry of Science and Technology under the project TIC2002-01846. Part of the results of this work were presented at the 19th Congress of the International Commission for Optics, Firenze, Italy, 25-31 August 2002 [13].

\section{References}

[1] Mandelbrot B B 1982 The Fractal Geometry of Nature (San Francisco, CA: Freeman)

[2] Berry M V 1979 J. Phys. A: Math. Gen. 12781

[3] Allian C and Cloitre M 1986 Phys. Rev. B 333563

[4] Jaggard D L and Sun X 1990 J. Opt. Soc. A 71131

[5] Sakurada Y, Uozumi J and Asakura T 1994 Pure Appl. Opt. 3 371

[6] Uozumi J and Asakura T 1994 Current Trends in Optics ed J C Dainty (Cambridge: Academic) pp 83-94

[7] Alieva T and Agulló López F 1996 Opt. Commun. 125267

[8] Lehman M 2001 Opt. Commun. 19511

[9] Berry M V and Klein S 1996 J. Mod. Opt. 432139

[10] Karman G P and Woerdman J P 2001 Opt. Lett. 231909

[11] Alieva T, Bastiaans M J and Barbé A 1999 Proc. ICASSP (Phoenix, USA) vol 3 p 1605

[12] Alieva T and Bastiaans M J 1999 Proc. Conf. on Fractals in Engineering (Delft, The Netherlands) (Rocquencourt, France: INRIA) p 43

[13] Alieva T and Calvo M L 2002 Proc. 19th Congr. ICO, Optics for the Quality of Life (Firenze, Italy) ed A Consortini and G Righini Proc. SPIE 4829 p 337 